\title{
On the nature of the phase transition in the three-dimensional random field Ising model
}

\author{
Vik.S. Dotsenko \\ LPTMC, Université Paris VI, 4 place Jussieu, 75252 Paris, France \\ L.D.Landau Institute for Theoretical Physics, 117940 Moscow, Russia
}

\begin{abstract}
A brief survey of the theoretical, numerical and experimental studies of the random field Ising model during last three decades is given. Nature of the phase transition in the three-dimensional RFIM with Gaussian random fields is discussed. Using simple scaling arguments it is shown that if the strength of the random fields is not too small (bigger than a certain threshold value) the finite temperature phase transition in this system is equivalent to the low-temperature order-disorder transition which takes place at variations of the strength of the random fields. Detailed study of the zero-temperature phase transition in terms of simple probabilistic arguments and modified mean-field approach (which take into account nearest-neighbors spin-spin correlations) is given. It is shown that if all thermally activated processes are suppressed the ferromagnetic order parameter $m(h)$ as the function of the strength $h$ of the random fields becomes history dependent. In particular, the behavior of the magnetization curves $m(h)$ for increasing and for decreasing $h$ reveals the hysteresis loop.
\end{abstract}

Key words:Quenched disorder, random fields, mean-field, hysteresis.

\section{Introduction}

The thermodynamic properties of the random field Ising model (RFIM) remains the controversial issue during more that 30 years now (for reviews see e.g. $[1,2]$ ). Traditionally there are exist three somewhat independent mainstreams of reseach: theoretical, numerical and experimental. Inside each of them, as well as between any of them, one finds broad divergences in the obtained results and in their interpretations. The main issue of this controversy is the structure of the phase diagram of the system: what kind of phase transitions and which thermodynamic phases are present there?

The random field model has been proposed originally by Larkin [3] to model the defect pining of vortices in sumerconductors. The most simple version of the this model for systems with discrete Ising symmetry, the random field Ising model (RFIM), can be described in terms of the Hamiltonian

$$
H=-\sum_{<i, j>}^{N} \sigma_{i} \sigma_{j}-\sum_{i} h_{i} \sigma_{i}
$$

where the Ising spins $\left\{\sigma_{i}= \pm 1\right\}$ are placed in the vertices of a D-dimensional lattice with the ferromagnetic interactions between the nearest neighbors, and quenched random fields $\left\{h_{i}\right\}$ are described either by the symmetric Gaussian distribution with $\left\langle h_{i} h_{j}\right\rangle=\delta_{i j} h^{2}$, or by the binary distribution in which the fields are taking two values $\pm h$ with independent probabilities at each site.

\section{$1.1 \quad$ Theory}

The first round of controversies in the studies of RFIM was about its lower critical dimension. According to simple energy ballace physical arguments by Imry and Ma [4] it should be expected that the dimensions $D_{c}$ above which the ferromagnetic ground state is stable at low temperatures (it is called the lower critical dimension) must be $D_{c}=2$ (unlike the pure Ising systems where $D_{c}=1$ ). Later, the existence of the ferromagnetic long range order in the 3D RFIM has been confirmed by a rigorous proof [5]. On the other hand, a perturbative renormalization group study of the phase transition demonstrates the so called phenomenon of a dimensional reduction, such that the critical exponents of the random field system in the dimension D appear to be the same as those of the ferromagnetic system without random fields in the dimension d=D-2 [6], which implies that the lower critical dimension of the random field Ising model must be equal to 3, 
in contradiction with the rigorous results. Actually, the procedure of summation of the leading large scale divergences assumes that the Hamiltonian has only one minimum. However, one can easily see that as soon as the temperature is close enough to the putative critical point (as well as in the whole low temperature region), there are local values of the magnetic fields for which the free energy has more than one minimum $[7,8]$, and therefore the dimensional reduction is not grounded. Thus, the above arguments have settled the controversy about the lower critical dimension of the random field Ising model in favor of the value $D_{c}=2$.

Phase diagram in the $(\mathrm{h}, \mathrm{T})$ plane for the RF Ising system has been first derived in terms of simple mean-field theory for the model with infinite-range ferromagnetic interactions (which corresponds to infinite space dimensionality) with the Gaussian distribution of random fields [9]. In this system the ferromagnetic phase is separated from the paramagnetic phase by a line $T(h)$ of the second order phase transition which monotonically decreases with $h$ from the point $T_{c}(0)$ of the pure system, down to the ending point $T=0$ at $h=h_{c}$. On the other hand, for the bimodal distribution [10] one finds that the line $T_{c}(h)$ contains a tricritical point $\left(T_{*}, h_{*}\right)$, such that at $h>h_{*}$ the phase transition becomes the first order. Later on a simple scaling description of the RFIM phase transition in finite dimensions has been developed assuming that it is of the second order, and that it is controlled by the zero-temperature fixed point [11].

As another extreme case, instead of the Ising spins, one can study the $m$-component vector spin system (the Ising model corresponds to $m=2$ ) with Gaussian random fields. It can be shown [12] that in terms of the replica field theory in the limit $m \rightarrow \infty$ the statistics of interfaces in this system is described by the solution with broken replica symmetry (similar to that in spin glasses [13]) which is consistent with the low critical dimension $d_{l}=2$. Later on in the same limit $m \rightarrow \infty$ it has been demonstrated [14] the instability of the replica symmetric state and the onset of the replica symmetry breaking scenario. In terms of this approach it has been argued that in the phase diagram of the three-dimensional RFIM the the paramagnetic and ferromagnetic phases are separated by the glassy phase in which the replica symmetry is broken [15].

An independent study of the $\phi^{4}$ replica field theory (which in the critical region is believed to describe RFIM) in terms of the Legendre transforms technique and the virial-like relations also shows the presence of the intermediate RSB glassy phase below dimensions $D=6$ [16]. Further renormalization-group (RG) study of this field theory in dimensions $D<6$ revealed no stable fixed points signalling the nonset of the replica symmetry breaking [17].

Another line of theoretical studies concerns the non-perturbative thermodynamic states which are apparently missing in the usual RG calculations and which may appear to be quite relevant for the nature of the phase transition in RFIM. The importance of the non-perturbative phenomena has been noted already long time ago $[7,18,19]$. In physical terms, the non-perturbative configurations are rare spatial regions with "flipped" (opposite to the background) magnetizations, and in terms of the replica field theory they are described by localized in space finite energy instanton configurations in which the replica symmetry is broken. In the recent investigations a systematic approach for the calculation of the non-perturbative contributions has been developed [21] and it has been shown that from the point of view of their relevance the dimension $D=3$ turns out to be marginal: formally these degrees of freedom produce finite (non-analytic in the strength of the field $h$ ) contribution only in dimensions $D \leq 3[22]$.

Finally, the construction of an alternative approach, the functional renormalization group, which is supposed to take into account all non-perturbative degrees of freedom for the whole class of the random-field $O(N)$ spin model is under way these very days [23]

\section{$1.2 \quad$ Numerics}

Numerical investigations of the phase transition in RFIM reveal broad controversy in the obtained results and in particular in their interpretation. The main point of the disagreements is about the nature if the phase transition: is it continuous, or is it of first order with finite jump of the order parameter.

Originally, the analysis of the high-temperature series expansion for the RFIM with the Gaussian distribution of random fields [24] indicated on the existence of a fluctuation-driven first order phase transition at sufficiently strong disorder below four dimensions (which suggests the existence of a tricritical point in the three-dimensional system). However more recent study indicated on the continuous transition, at leas for weak disorder [25].

First extensive Monte Carlo (MC) numerical simulations for the 3D RFIM have been carried out by Young and Nauenberg [26], who reported that the transition may be first order. On the other hand, the numerical study by Ogielski and Huse [27], and by Ogielski [28] shows that the transition may be second order. More recent Monte Carlo simulations [29] can also been interpreted as describing a continuous transition but with 
a finite jump in the magnetization. However, later extensive MC calculations [30] for the energy and the magnetization distributions suggest a first order transition.

The zero-temperature studies play an important role in understanding the nature of the phase transition in the RFIM. According to the zero temperature fixed point hypothesis, the transitions which take place at $T=0$ and at $T \neq 0$ are in the same universality class. First zero-temperature Migdal-Kadanoff renormalization-group calculations indicated a continuous transition which is characterized by very small value of the magnetization critical exponent $(\beta \simeq 0.02)$ [31]. Similar results were obtained in the finite temperature renormalization group study [32]. On the other hand, some of more recent zero-temperature finite size scaling calculations [33] have demonstrated the existence of a first order phase transition, although most of the them $[34,35]$ are in favor of the continuous transition. Moreover, in one of the most recent calculations it has been demonstrated that for a given realization of disorder the ground states $(T=0)$ and thermal states $(T \neq 0)$ near the critical line are strongly correlated, which may be interpreted as the concrete manifestation of the zero temperature fixed point scenario [36]. The most precise results obtained by A.A.Middleton and D.S.Fisher [35] are clearly consistent with the existence of a single phase transition (no intermediate spin-glass phase has been observed) and it is characterized by very small value of the magnetization critical exponent $\beta=0.017$.

Others numerical studies of the phase diagram of the RFIM in the plane $(T, h)[37,38]$, also find no glassy phase (with or without replica symmetry breaking) which would separate paramagnetic and ferromagnetic phases. On the other hand, for the bimodal random field distributions a discontinuity in the magnetization at the phase transition line is observed [38].

Finally, the numerical simulations of the diluted antiferromagnet in a field (DAFF) designed to imitate the random field magnetic systems which are studied experimentally (see below), shows that in equilibrium, the transition to the LRO state may be first order [39,40], and moreover it is claimed that at sufficiently strong dilution there is first order phase transition to a spin glass state [41]

To conclude this brief survey of numerical studies, it should be noted that all numerical simulations in RFIM are impeded by the dramatic slowing down when approaching a putative phase transition, which very often makes very difficult to interpret the obtained results. Besides, the delicate point in all these studies is that the order parameter critical exponent $\beta$ turns out to be very small, so it is very difficult to distinguish the second order transition (for which the order parameter continuously goes to zero at $T_{c}$ ) from the first order (when the order parameter has a finite jump at $T_{c}$ ). On top of that, recent numerical study of the critical region of the 3D RFIM indicates that the situation could be even more sophisticated due to absence of self-averaging of the correlation length, of the specific heat, and maybe of some others thermodynamical quantities [42].

\subsection{Experiments}

From the point of view of experimental investigations an important theoretical breakthrough occurred when it has been realized that RFIM can be generated in diluted Ising antiferromagnets by application of a uniform external magnetic field [43]. This has opened the way for experimental studies of RFIM, and in particular for the investigation of the phase transition.

In the very first experimental studies of the RFIM performed in the diluted antiferromagnets $C o_{x} Z n_{1-x} F_{2}$ it has been claimed that even the smallest magnetic fields destroy the long-range order at all temperatures in three dimensions [44].

First convincing experimental demonstrations of the existence of the phase transition in the 3D random field antiferromagnet $F e_{x} Z n_{1-x} F_{2}$ and its critical properties (assuming that it is of second order) has been reported in [45]. The extensive study of the critical properties of the system $F e_{0.6} Z n_{0.4} F_{2}$ for temperatures $T>T_{c}(h)$ has been done in [46].

In fact, the behavior observed near the critical point depends on the field and temperature procedures used in the measurements. In the field cooled (FC) samples the transition appears to be rounded, and at low temperatures the system freezes in the metastable domain state. However, if the sample is cooled in the zero field (ZFC), the transition appears to be much sharper, and rounded only by slow dynamic effects [2].

On the other hand, in another experimental study of the 3D RFIM (for weakly anisotropic $M n_{0.5} Z n_{0.5} F_{2}$ ) [47] it has been observed that when approaching the phase transition the correlation length reaches a finite size, at which point it was assumed that further approach towards a putative second order transition is interrumpted by occurrence of a first order transition.

The most slippery issue of the experimental studies of the phase transition in the RFIM is the measurement 
of the static critical behavior of the staggered magnetization. Similarly to the numerical studies, the fact that critical exponent $\beta$ is experimentally very small (e.g. according to [48] $\beta \simeq 1 / 8$ or less, according to [49] $\beta \simeq 0.16$ ) makes it very difficult to work out any definite conclusion about the nature of the observed phase transition.

In the studies of the diluted antiferromagnet $M n_{0.75} Z n_{0.25} F_{2}$ the field-cooled data were interpreted as the evidence for an equilibrium second-order transition at $T_{F C}$, while the zero-field cooled data were interpreted as non-equilibrium trompe l'oeil transition in which the long-range order diminishes continuously at $T_{Z F C}>$ $T_{F C}$, while the correlation length only reaches a finite maximum value at a temperature between $T_{Z F C}$ and $T_{F C}$ [50]. Similar behavior was observed in $F e_{0.5} Z n_{0.5} F_{2}$ [51]. On the other hand, all these results can be explained as the mean-field first-order transition broadened by the cluster-flipping mechanism [52].

In the study of the random field antiferromagnet $F e_{0.5} Z n_{0.5} F_{2}$ a notable broadening of the phase transition region has been observed, so that the data may be described by the Gaussian distribution of effective transition temperatures with the width which scales with the strength of the applied field as $h^{2}$ [53]. Besides, the apparent critical behavior observed in these measuremets represented a continuous evolution from metastable behavior towards equilibrium behavior. Moreover, according to the point of view of Birgeneau [54], so far no true equilibrium phase transition has been observed in RFIM systems. Nevertheless, in the recent study of high-magnetic concentration Ising antiferromagnet $F e_{0.93} Z n_{0.07} F_{2}$ (which does not exhibit the severe critical scattering hysteresis [55]) it is claimed that in terms of general scaling assumptions the critical scattering analysis allows to reveal equilibrium critical behavior and to obtain the corresponding critical exponents [56] (in particular the specific heat critical behavior is well fitted by the logarithmic singularity which corresponds to $\alpha=0$ [57]).

In conclusion of this brief historic review one can note that the situation with the phase transition in the three-dimensional RFIM remains far from being clear. Theorists are mostly interested in their own problems (like $1 / m$ expansion, or RSB in dimensions close to 6 , or two-loop approximation for $O(N)$ sigma model with the number of spin components close to $N_{c} \simeq 2.835$ ), which are rather far from the original system, while people doing numerics and in particular experiments are facing realities full of numerous secondary and accessory effects which very often overshadow the main physical phenomena.

It is evident that one can not hope to find the exact solution for this problem, and therefore some kind of simplifications and approximations are unavoidable. In this paper, which is mostly devoted to the lowtemperature part of the phase diagram, it will be supposed that: (a) the finite temperature thermodynamical properties of the three-dimensional RFIM can be described in terms of the continuous Ginzburg-Landau $\phi^{4}$ field theory; (b) we can neglect the presence of randomness in the spin-spin interactions; and (c) in the study of the zero-temperature phase transition (at variation of the strength of the random fields) all thermally activated processes are suppressed.

The above reservations are rather essential.

(a) Ginzburg-Landau field theory. We all believe in the universality, and therefore if we admit that the phase transition in a given system is of the second order, then it is generally accepted that its critical properties can be described in terms of the corresponding Ginzburg-Landau Hamiltonian (with proper symmetry properties). However, if the system is considered at a finite distance from the putative critical point (which is the case in the present paper) where the correlation length is finite, then the relevance of the continuous limit Ginzburg-Landau Hamiltonian for the original lattice Ising system becomes much less evident.

(b) Randomness in the spin-spin interactions. Apart from the numerical investigations of DAFF $[39,41]$, the effects of randomness in the spin-spin interactions is practically never taken into account in theoretical studies (there are enough headache due to random fields...). However, it is well known that the presence of this type of randomness (without random fields) may change the critical properties of a magnetic system, and in particular it definitely does in the three-dimensional Ising model. Moreover, in some statistical systems (e.g. in the Potts model) the presence of such disorder may turn the first-order transition (of the pure system) into the second order one [58]. Therefore, it we admit for the moment that the phase transition in the "pure" (without randomness in spin-spin interactions) 3D RFIM is of the first order, it does not guarantee that in the corresponding experimental realizations of this system (where the randomness in the spin-spin interactions is inevitable) the phase transition would not turn into the second order one.

(c) Thermal activations. In the studies of the zero-temperature properties of the system as the function of the effective strength of the random fields $h$, one can consider two types of procedures. In the first one it 
is assumed that at any given $h$ the system is at the ground state (which, at a given realization of the random fields, is assumed to be unique). In this case the variation of $h$ to a new value $h^{\prime}$ means that either we admit that the temperature is actually slightly non-zero, and the system passes to a new ground state configuration (corresponding to $h^{\prime}$ ) via thermally activated flips, or at each variation of $h$ the ground state configuration is achieved by cooling down the system (at fixed $h^{\prime}$ ) from the high-temperature disordered state.

The alternative procedure of the variation of $h$ is essentially different. Here one has to fix the starting value of $h($ e.g. $h=0)$, and then at any variation of $h$ the spins are just following the directions of their local fields (which consist of the contributions due to the regular ferromagnetic interactions with the nearest neighbors plus the local value of the random field). In this procedure all thermal "jumps over energy barriers" are prohibited, and therefore, most probably, due to the variation of $h$ the system will get stuck in one of the metastable states.

Of course, from the experimental point of view none of these two procedures can be implemented in the pure form. The first one (qualitatively, it corresponds to the field cooled (FC) measurements) requires very long (formally infinite) waiting time to reach the thermal equilibrium at any variation of $h$, while the second one (it corresponds to the zero field cooled (ZFC) measurements) requires to suppress all thermally activated processes which can be achieved only if the temperature is exactly equal to zero.

In the next Section using simple physical arguments it will be shown that if the strength of the random fields is not too small then there exists a region of parameters where the critical fluctuations are irrelevant, and therefore the thermodynamic properties of the system can be described in terms of the saddle-point equations of the corresponding (random) Ginzburg-Landau Hamiltonian. It is in this region that the ferromagnetic order breaks down. Moreover, using simple scaling arguments it will be shown that in this case the finite temperature phase transition in this system is equivalent to the low-temperature phase transition at variation of the strength of the random fields.

In Section 3 the detailed study of the zero-temperature order-disorder phase transition will be done in terms of the discrete Ising model on the three-dimensional cubic lattice. Here we propose a modified (slightly improved) version of the mean-field approach which takes into account nearest-neighbors two-spins correlations. Then, considering the two regimes: increasing and decreasing strength of the random fields, and supposing that all thermally activated spin flips are suppressed, it will be demonstrated that the magnetization curve $m(h)$ reveals the hysteresis loop. In particular, it is characterized by two different critical points $h_{c}^{(-)}<h_{c}^{(+)}$, where $h_{c}^{(-)}$is the strength of the random fields at which $m(h)$ becomes non-zero at decreasing $h$, and $h_{c}^{(+)}$is the one at which $m(h)$ becomes zero at increasing $h$.

\section{Heuristic arguments}

For the sake of generality, before coming down to the dimension three, first, let us consider the continuous version of the $D$-dimensional Ising model in terms of the scalar field Ginzburg-Landau Hamiltonian:

$$
H[\phi(\mathbf{x}), h(\mathbf{x})]=\int d^{D} x\left[\frac{1}{2}(\nabla \phi(\mathbf{x}))^{2}-\frac{1}{2} \tau \phi^{2}(\mathbf{x})+\frac{1}{4} g \phi^{4}(\mathbf{x})-h(\mathbf{x}) \phi(\mathbf{x})\right]
$$

Here $\tau=\left(T_{c}-T\right) / T_{c}(\tau \ll 1)$ is the reduced temperature parameter (for simplicity in what follows it will be supposed that $\left.T_{c}=1\right)$. According to this definition, positive values of $\tau$ correspond to the low-temperature ferromagnetic state. Random fields $h(\mathbf{x})$ are described by the symmetric Gaussian distribution,

$$
P[h(\mathbf{x})]=p_{0} \exp \left(-\frac{1}{2 h^{2}} \int d^{D} x h^{2}(\mathbf{x})\right)
$$

where $h$ is the parameter which describes the strength of the random field, and $p_{0}$ is an irrelevant normalization constant. For a given realization of the random fields the partition function of this system is obtained by the integration over all configurations of the scalar fields $\phi(\mathbf{x})$

$$
Z[h(\mathbf{x})]=\int \mathcal{D} \phi(\mathbf{x}) \exp (-H[\phi(\mathbf{x}), h(\mathbf{x})])
$$


It is well known that if we consider such system at temperatures not too close to the critical point (so that the parameter $\tau$ is not too small) the thermal critical fluctuations are irrelevant, and the leading contributions to the partition function, eq.(4), are coming from the minima of the Hamiltonian, eq.(2), described by the saddle-point equation

$$
-\Delta \phi(\mathbf{x})-\tau \phi(\mathbf{x})+g \phi^{3}(\mathbf{x})=h(\mathbf{x})
$$

In the pure system (at $h(\mathbf{x})=0$ ) the restriction on the value of the parameter $\tau$ is given by the GinzburgLandau condition

$$
\tau \gg g^{2 /(4-D)} \equiv \tau_{G L}
$$

It is clear that this requirement makes sense only if the coupling parameter $g$ is sufficiently small. Of course, formally for the three-dimensional Hamiltonian, eq.(2), considered as the continuous limit representation of the original Ising model this is not true (unlike the corresponding systems in dimensions close to four, where the effective (renormalized) value of the coupling parameter $g \sim \epsilon=(4-D) \ll 1)$. Nevertheless, here we are going to consider the random field Ginzburg-Landau theory, eq.(2) in which the coupling parameter $g$ is assumed to be sufficiently small, hoping that at the qualitative level the behavior of the system is not so much sensitive to the actual value of this parameter.

The only relevant spatial scale in the system described by the Hamiltonian (2) is the correlation length $R_{c}(\tau)$ which under condition (6) has the scaling

$$
R_{c}(\tau) \sim \tau^{-1 / 2}
$$

In the absence of the random fields the ferromagnetic ground state of the system is characterized by the homogeneous configuration $\phi_{0}=\sqrt{\tau / g}$. To study the effects produced by the random fields on the ferromagnetic state of the system let us perform the following space and fields rescaling

$$
\phi(\mathbf{x})=\left(\frac{\tau}{g}\right)^{1 / 2} \varphi\left(\mathbf{x} / R_{c}\right)
$$

In terms of the new fields $\varphi(\mathbf{z})$, where $\mathbf{z} \equiv \mathbf{x} / R_{c}=\tau^{1 / 2} \mathbf{x}$, the system is described by the rescaled Hamiltonian

$$
H[\varphi(\mathbf{z}), \tilde{h}(\mathbf{z})]=\frac{\tau^{\frac{(4-D)}{2}}}{g} \int d^{D} z\left[\frac{1}{2}(\nabla \varphi(\mathbf{z}))^{2}-\frac{1}{2} \varphi^{2}(\mathbf{z})+\frac{1}{4} \varphi^{4}(\mathbf{z})-\sqrt{\frac{g}{\tau^{3}}} \tilde{h}(\mathbf{z}) \varphi(\mathbf{z})\right]
$$

where the rescaled random fields

$$
\tilde{h}\left(\mathbf{x} / R_{c}\right)=R_{c}^{-D} \int_{\left|\mathbf{x}^{\prime}-\mathbf{x}\right|<R_{c}} d^{D} x^{\prime} h\left(\mathbf{x}^{\prime}\right)
$$

are described by the distribution function

$$
P[\tilde{h}(\mathbf{z})]=\tilde{p}_{0} \exp \left(-\frac{1}{2 h^{2} \tau^{D / 2}} \int d^{D} z \tilde{h}^{2}(\mathbf{z})\right)
$$

(note that the integration here involves the ultraviolet cutoff lengthscale equal to one). Redefining the random fields again

$$
\tilde{h}(\mathbf{z})=\sqrt{\frac{\tau^{3}}{g}} \xi(\mathbf{z})
$$

instead of eq.(4) we obtain the partition function

$$
Z[\xi(\mathbf{z})]=\int \mathcal{D} \varphi(\mathbf{z}) \exp (-\tilde{\beta} \tilde{H}[\varphi(\mathbf{z}), \xi(\mathbf{z})])
$$

which is controlled by the effective "inverse temperature" 


$$
\tilde{\beta} \equiv\left(\frac{\tau}{\tau_{G L}}\right)^{\frac{(4-D)}{2}} \gg 1
$$

( $\tau_{G L}$ is the Ginzburg-Landau temperature, eq(6)) and which is defined by the new effective Hamiltonian

$$
H[\varphi(\mathbf{z}), \xi(\mathbf{z})]=\int d^{D} z\left[\frac{1}{2}(\nabla \varphi(\mathbf{z}))^{2}-\frac{1}{2} \varphi^{2}(\mathbf{z})+\frac{1}{4} \varphi^{4}(\mathbf{z})-\xi(\mathbf{z}) \varphi(\mathbf{z})\right]
$$

which contains no parameters. The new random fields $\xi(\mathbf{z})$ are described by the Gaussian distribution

$$
P[\xi(\mathbf{z})]=p_{0} \exp \left(-\frac{1}{2 \lambda^{2}} \int d^{D} x \xi^{2}(\mathbf{z})\right),
$$

characterized by the mean square value

$$
\lambda^{2}=\frac{\left(g h^{2}\right)}{\tau^{(6-D) / 2}}
$$

The ground state configurations in terms of the new fields $\varphi(\mathbf{z})$ are defined by the saddle-point equation

$$
-\Delta \varphi(\mathbf{z})-\varphi(\mathbf{z})+\varphi^{3}(\mathbf{z})=\xi(\mathbf{z})
$$

In terms of the new fields, $\varphi(\mathbf{z})$ and $\xi(\mathbf{z})$, the transition from the ordered (ferromagnetic) to the disordered (paramagnetic) state looks as follows. In the absence of the random fields $(\xi \equiv 0)$ the ground state is given by the trivial ferromagnetic solution $\varphi_{0}(\mathbf{z})=1$ (or $\varphi_{0}(\mathbf{z})=-1$ ). The presence of weak random fields (at $\lambda \ll 1$ ) introduces only small perturbations to this solution. However, if we increase the effective strength of $\xi(\mathbf{z})$, which is controlled by the parameter $\lambda$, eq.(17), the ferromagnetic configuration is getting more and more perturbed, and finally, at at a certain critical value $\lambda_{c}$ the ferromagnetic ordering is destroyed.

One can note two important points in the above scenario:

First. According eq.(17), for fixed value of the parameter $h$ (which is the strength of the original random fields $h(\mathbf{x})$ ), the effective strength of the random fields $\xi(\mathbf{z})$ is controlled by the temperature parameter $\tau$. According to its definition $(\tau=(1-T))$, increasing the temperature $T$ of the system means decreasing $\tau$, which in turn, produces increasing of the parameter $\lambda$ In other words, variations in temperature turn into variations of the effective strength of the random fields.

Second. According to the saddle-point equation (18) the critical value of $\lambda_{c}$ is of the order of one. This means that the transition takes place at

$$
\tau \sim \tau_{c}=\left(g h^{2}\right)^{\frac{2}{(6-D)}}
$$

At this point the value of the effective "inverse temperature" parameter $\tilde{\beta}$, eq.(14) is

$$
\tilde{\beta}_{c}=\left(\frac{h^{2}}{g^{2 /(4-D)}}\right)^{\frac{(4-D)}{(6-D)}}
$$

Thus, if the strength of random fields is not too small:

$$
h \gg h_{*}(g)=g^{\frac{1}{(4-D)}}
$$

at dimensions $D<4$ in the vicinity of the phase transition (when $\tau \sim \tau_{c}$ ) we have

$$
\tilde{\beta} \sim \tilde{\beta}_{c}=\left(\frac{h}{h_{*}(g)}\right)^{\frac{2(4-D)}{(6-D)}} \gg 1
$$

In other words, in terms of the representation, eqs.(13)-(17), the original ferromagnetic-paramagnetic phase transition at any point of the critical curve $h_{c}(T)$ on the left of the point $\left(h_{*}, T_{*}\right)$ (Figure 1) is equivalent to the low-temperature order-disorder transition (far left extreme of the curve $h_{c}(T)$ ) at variation of the strength of the random fields. In particular, in the dimension $D=3$, 


$$
\begin{aligned}
& \tau_{G L}=g^{2} \\
& \tau_{c}=\left(g h^{2}\right)^{2 / 3} \\
& \tilde{\beta}_{c}=\left(\frac{h}{g}\right)^{2 / 3} \\
& h_{*}(g)=g
\end{aligned}
$$

The conditions $\tau_{c} \gg \tau_{G L}, \tilde{\beta}_{c} \gg 1$ and $h \gg h_{*}$ discussed above are automatically satisfied by the only restriction on the strength of the random fields,

$$
h \gg g
$$

In the next section to understand the nature of the low temperature phase transition, we are going to consider its extreme version in the zero temperature limit. In this case it is natural to consider the original discrete Ising model on a lattice instead of its continuous limit representation.

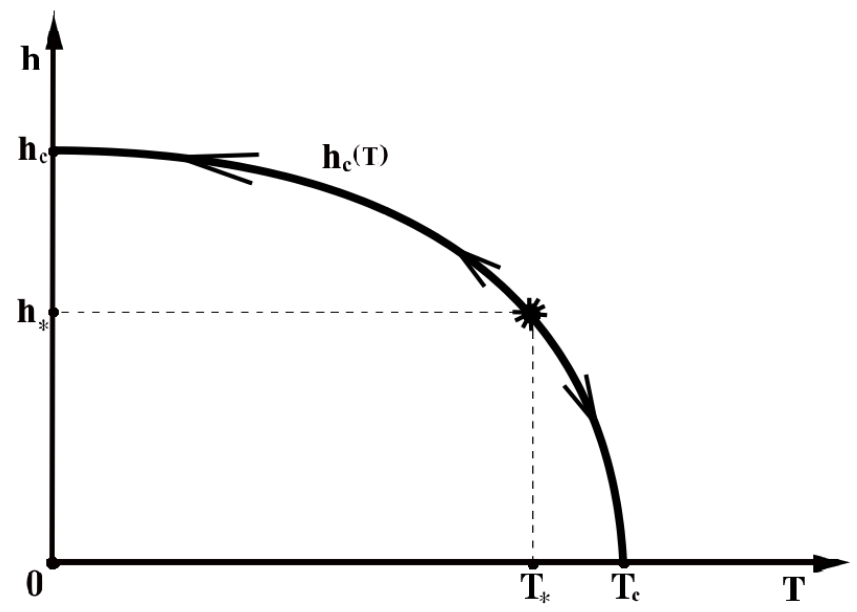

Figure 1: Qualitative phase diagram of the random field Ising model

\section{Zero-temperature phase transition}

Let us consider the three-dimensional random field ferromagnetic Ising model on the cubic lattice with the Hamiltonian

$$
H=-\frac{1}{12} \sum_{<j \neq j>}^{N} \sigma_{i} \sigma_{j}-\sum_{i}^{N} h_{i} \sigma_{i}
$$

Here $<j \neq j>$ denotes the pairs of nearest neighbors, and the random fields $h_{i}$ are described by the independent Gaussian distributions

$$
W_{h}\left[h_{1}, h_{2}, \ldots, h_{N}\right]=\prod_{i=1}^{N} \mathcal{P}_{h}\left(h_{i}\right)
$$

where

$$
\mathcal{P}_{h}\left(h_{i}\right)=\frac{1}{\sqrt{2 \pi h^{2}}} \exp \left(-\frac{h_{i}^{2}}{2 h^{2}}\right)
$$


The ground state spin configuration of this system is defined by the set of $N$ the conditions

$$
\sigma_{i}=\operatorname{Sign}\left(\frac{1}{6} \sum_{\alpha_{i}=1}^{6} \sigma_{\alpha_{i}}+h_{i}\right)
$$

where for every site $i$ the summation goes over its 6 nearest neighbors.

First, let us discuss at the qualitative level what is going on if we increase the parameter $h$ from zero to large values. At $h=0$ the state of the system is ferromagnetic: the solution of the above equations (28) is trivial, $\sigma_{i}=+1$ (of course, there is another equivalent solution $\sigma_{i}=-1$, but in what follows we will suppose that in the ferromagnetic state the spins are directed "up"). At non-zero $h \ll 1$ almost all the spins are directed "up", but in rare cases, when at a given site $i$ the value of the local field is sufficiently negative, $h_{i}<-1$, the $i$-th spin is flipped "down". According to eq.(27), the probability of this event (which is equal to the concentration of the negative spins) is

$$
\rho(h \ll 1)=\int_{-\infty}^{-1} d x \mathcal{P}_{h}(x)=\frac{1}{2} \operatorname{Erfc}\left(\frac{1}{\sqrt{2} h}\right)
$$

where

$$
\operatorname{Erfc}(z) \equiv \frac{2}{\sqrt{\pi}} \int_{z}^{+\infty} d t \exp \left(-t^{2}\right)
$$

is the complementary error function (which exponentially goes to zero at $z \gg 1$ ).

Note that in the above consideration we have neglected the configurations in which the flipped down spins appear to be the nearest neighbors. It is clear that at small $h$ the concentration $\rho(h)$ is exponentially small and therefore the probability of these events is negligible. However, when increasing the value of the parameter $h$ the probability to find two or more neighboring "down" spins becomes non-small, and it is due to these configuration that the situation becomes rather sophisticated. First of all, one can note that if at neighboring sites the values of the random fields appear to be non small (and negative), then the stable spin configurations can become ambiguous. As an example let us consider two neighboring sites $i$ and $i+1$ (surrounded by the spins which all are directed "up") with the values of the random fields $-1<h_{i}<-2 / 3$ and $-1<h_{i+1}<-2 / 3$. One can easily see that in this case both the configuration $\sigma_{i}=\sigma_{i+1}=+1$ and the configuration $\sigma_{i}=\sigma_{i+1}=-1$ satisfy the stability conditions eq.(28). It is obvious that one could find similar phenomena in clusters consisting of larger number of spins.

The effects of the ambiguity of the stationary spin configurations due to the presence of the random fields and their (non-perturbative) contributions to the finite-temperature thermodynamics have been discussed earlier in terms of the continuous Ginzburg-Landau representation of the RFIM [22]. Here we will describe the consequences of the ambiguity of the stationary states for the phase transition at the zero temperatures. At the qualitative level it is tempting to suggest that the presence of such kind of phenomena could be the origin of the phase transition of the first order. Indeed, if at increasing the strength of the random field the system gets stuck at ferromagnetic configurations, an alternative disordered states could become energetically preferable before the ferromagnetic state would become unstable. Note that unlike the second-order phase transitions which are characterized by the divergence of the correlation length (and which require to find the way to study the system at large scales) the first-order phase transition is characterized by a finite value of the correlation length. For that reason one can hope that taking into account correlations only of the order of the lattice spacing could still give reasonable results.

\subsection{Mean-field approach}

First, as a matter of simple "warming up exercise", let us consider how the the zero-temperature phase transition looks like if we study it in terms of the ordinary mean-field approach (which neglects the spin-spin correlations at all).

Let us denote by $x$ the probability that a given spin $i$ points "up". Then the probability to be "down" is or course equal to $(1-x)$. In this case the ferromagnetic order parameter which is the global magnetization of the system is

$$
m=\frac{1}{N} \sum_{i}^{N} \sigma_{i}=\overline{\sigma_{i}}=2 x-1
$$




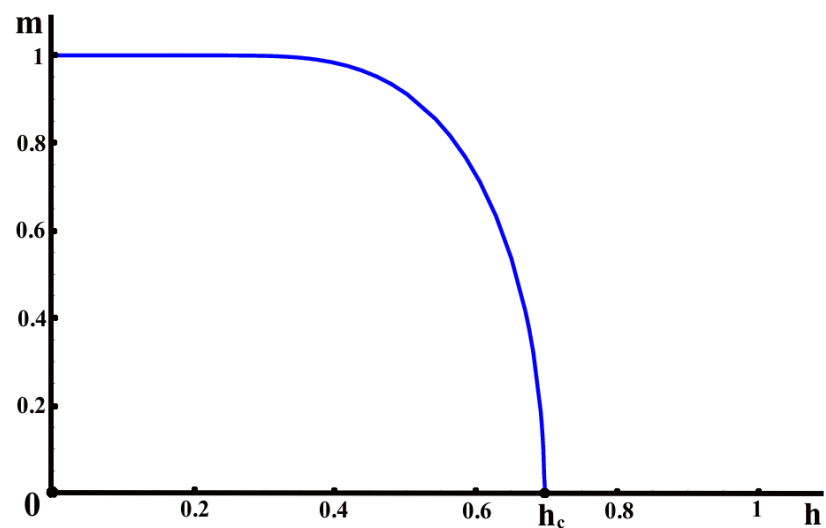

Figure 2: Mean field solution for the order ferromagnetic order parameter $m(h)\left(h_{c} \simeq 0.696\right)$

A given spin is surrounded by the six nearest neighbors. If all the neighbors are pointing "up" (the probability of this configuration is equal to $x^{6}$ ) then $\sigma_{i}=+1$ provided the local field $h_{i}>-1$. If one of the neighbors is "down" (the probability is $6 x^{5}(1-x)$ ), then $\sigma_{i}=+1$ provided the local field $h_{i}>-2 / 3$. If two of the neighbors are "down" (the probability is $\frac{6 \cdot 5}{2} x^{4}(1-x)^{2}$ ), then $\sigma_{i}=+1$ provided the local field $h_{i}>-1 / 3$. Etc. Collecting all these configurations one easily gets the following self-consistent equation for $x$ :

$$
\begin{aligned}
x & =x^{6} \cdot P_{6}(h)+6 x^{5}(1-x) \cdot P_{5}(h)+15 x^{4}(1-x)^{2} \cdot P_{4}(h)+20 x^{3}(1-x)^{3} \cdot P_{3}(h)+ \\
& +15 x^{2}(1-x)^{4} \cdot P_{2}(h)+6 x(1-x)^{5} \cdot P_{1}(h)+(1-x)^{6} \cdot P_{0}(h)
\end{aligned}
$$

Here we have introduced the notations

$$
\begin{aligned}
& P_{6}(h)=\int_{-1}^{\infty} d y \mathcal{P}_{h}(y)=1-\frac{1}{2} \operatorname{Erfc}\left(\frac{1}{\sqrt{2} h}\right) \\
& P_{5}(h)=\int_{-2 / 3}^{\infty} d y \mathcal{P}_{h}(y)=1-\frac{1}{2} \operatorname{Erfc}\left(\frac{2}{3 \sqrt{2} h}\right) \\
& P_{4}(h)=\int_{-1 / 3}^{\infty} d y \mathcal{P}_{h}(y)=1-\frac{1}{2} \operatorname{Erfc}\left(\frac{1}{3 \sqrt{2} h}\right) \\
& P_{3}(h)=\int_{0}^{\infty} d y \mathcal{P}_{h}(y)=\frac{1}{2} \\
& P_{2}(h)=\int_{1 / 3}^{\infty} d y \mathcal{P}_{h}(y)=\frac{1}{2} \operatorname{Erfc}\left(\frac{1}{3 \sqrt{2} h}\right) \\
& P_{1}(h)=\int_{2 / 3}^{\infty} d y \mathcal{P}_{h}(y)=\frac{1}{2} \operatorname{Erfc}\left(\frac{2}{3 \sqrt{2} h}\right) \\
& P_{0}(h)=\int_{1}^{\infty} d y \mathcal{P}_{h}(y)=\frac{1}{2} \operatorname{Erfc}\left(\frac{1}{\sqrt{2} h}\right)
\end{aligned}
$$

The solution of eq.(32) for the ferromagnetic order parameter is shown in Figure 2. We see that the phase transition from the ferromagnetic $(m \neq 0)$ to the paramagnetic $(m=0)$ state takes place at $h_{c} \simeq 0.696$. One can easily check that in the vicinity of the critical point, as $h \rightarrow h_{c}$, the the ferromagnetic order parameter vanishes according to the the scaling law:

$$
m(h) \sim\left(h_{c}-h\right)^{\beta}
$$

described by the usual mean-field critical exponent $\beta=0.5$ (see Figure 3). This, of course, is not surprising because if in eq.(32) we substitute $x=(m+1) / 2$, the resulting equation for $m$ (whatever complicated it may 


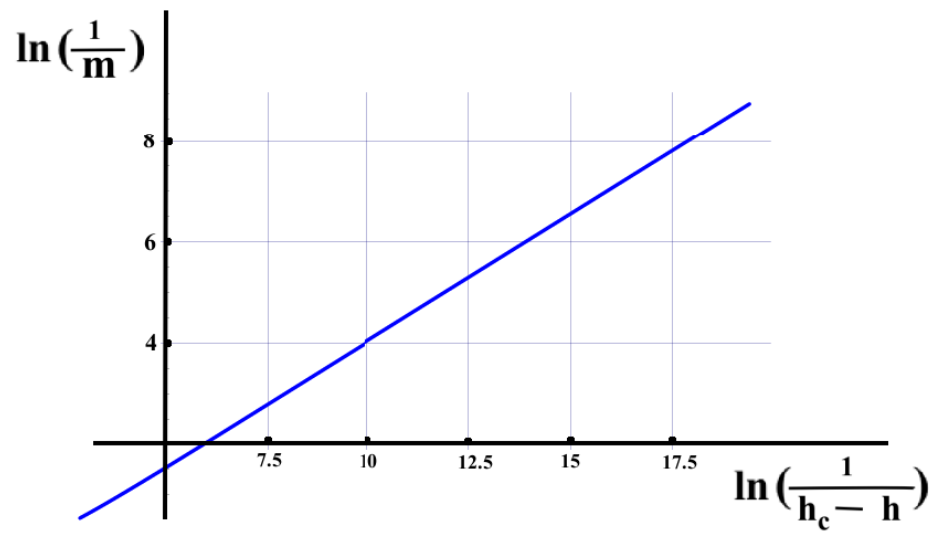

Figure 3: Log-log plot for the ferromagnetic order parameter in the vicinity of the critical point

look like) will be still the mean field equation for the order parameter, and its development in powers of small $m(h)$ in the vicinity of the critical point can not give anything else but mean-field critical exponent.

It is clear that the consideration presented above can not pretend to describe the true phase transition which takes place in the system under consideration. The reason for that is obvious: in such king of the mean-field calculations one misses all spin-spin correlations, which, as we have discussed in the beginning of this section, are crucial for the statistical properties of the present system.

Below we are going to modify the above approach so that it would take into account correlations between pairs of nearest neighbors spins. It turns out that even such rather limited improvement is sufficient to intoduce rather dramatic changes in the scenario of the phase transition.

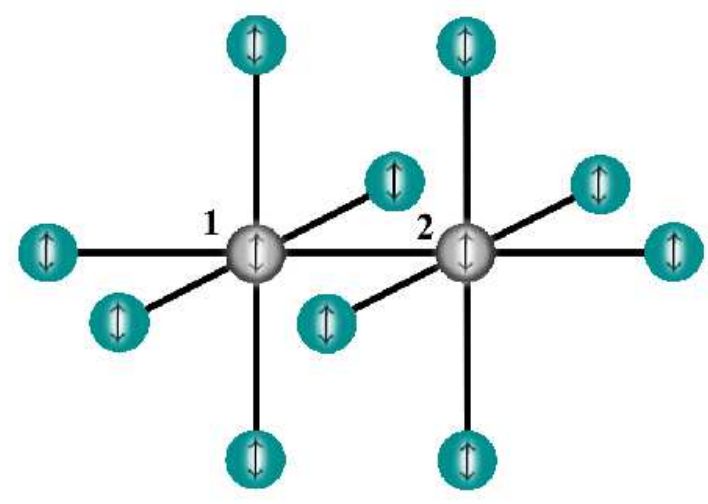

Figure 4: Two neighboring sites are surrounded by $(5+5)$ nearest neighbors

\section{$3.2 \quad$ Modified mean-field approach}

Let us consider a pair of nearest neighbor spins $\sigma_{1}$ and $\sigma_{2}$ and let us denote by $p(\uparrow \uparrow), p(\uparrow \downarrow), p(\downarrow \uparrow)$ and $p(\downarrow \downarrow)$ the probabilities of the $\left(\sigma_{1}, \sigma_{2}\right)$ configurations $(+1,+1),(+1,-1),(-1,+1)$ and $(-1,-1)$ correspondingly. These four probabilities are, of course, bounded by the condition $p(\uparrow \uparrow)+p(\uparrow \downarrow)+p(\downarrow \uparrow)+p(\downarrow \downarrow)=1$. As before, by $x$ we denote the probability for a given spin to be "up". One can easily see that

$$
x=p(\uparrow \uparrow)+p(\uparrow \downarrow)
$$


The two spins $\sigma_{1}$ and $\sigma_{2}$ are surrounded by $(5+5)$ neighbors (Figure 4), and their orientations are defined by the equations:

$$
\begin{aligned}
& \sigma_{1}=\operatorname{Sign}\left[\frac{1}{6}\left(\sum_{\alpha_{1}=1}^{5} \sigma_{\alpha_{1}}+\sigma_{2}\right)+h_{1}\right] \\
& \sigma_{2}=\operatorname{Sign}\left[\frac{1}{6}\left(\sum_{\alpha_{2}=1}^{5} \sigma_{\alpha_{2}}+\sigma_{1}\right)+h_{2}\right]
\end{aligned}
$$

where $\sigma_{\alpha_{1,2}}$ denote the neighbors of the spins $\sigma_{1,2}$.

The idea is to compute $p(\uparrow \uparrow)$ and $p(\uparrow \downarrow)$ by summing over all possible configurations of these neighboring spins (with the corresponding probabilities defined by the parameter $x$ ) and integrating over local fields $h_{1}$ and $h_{2}$ (with the probability distribution $\mathcal{P}_{h}\left(h_{1}\right) \mathcal{P}_{h}\left(h_{2}\right)$ ). Then, substituting $p(\uparrow \uparrow$ ) and $p(\uparrow \downarrow$ ) (which will be the functions of $x$ and $h$ ) into eq.(35), we will get a self-consistent equation for $x$. It turns out, however, that this program can not be implemented directly, because for every configuration of surrounding spins there are finite regions in the plane $\left(h_{1}, h_{2}\right)$, where the orientations of spins $\sigma_{1}$ and $\sigma_{2}$ are not uniquely defined by the given values of $h_{1}$ and $h_{2}$.
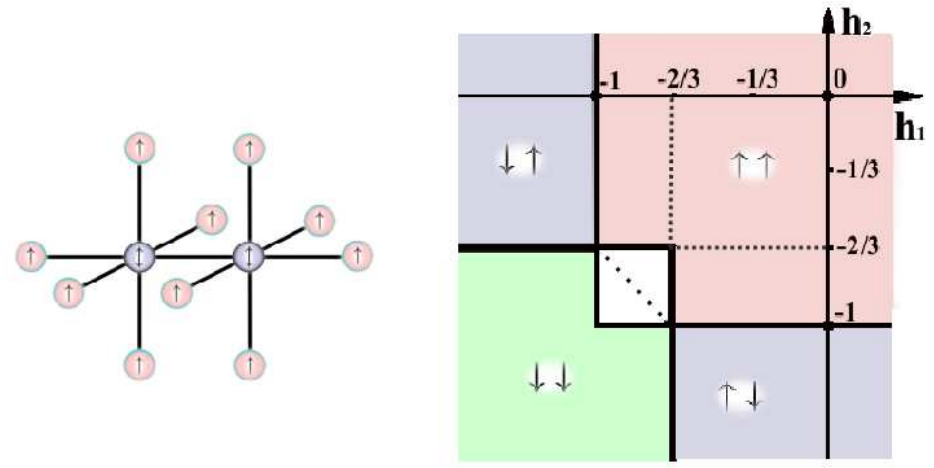

Figure 5: The structure diagram of the orientations of two neighboring spins for the case when all their $(5+5)$ nearest neighbors are directed "up"

As an example let us consider again the simplest situation when all ten neighboring spins are directed "up" (which has the probability $x^{10}$ ). In this case the condition to have both spins" up" is $h_{1}>-1$ and $h_{2}>-1$ (so that the corresponding contribution to $p(\uparrow \uparrow)$ must be given by the integration of $\mathcal{P}_{h}\left(h_{1}\right) \mathcal{P}_{h}\left(h_{2}\right)$ in the sector $\left.\left(h_{1}>-1, h_{2}>-1\right)\right)$. The contribution to the probability $p(\uparrow \downarrow)$ is given by the sector $\left(h_{1}>-2 / 3, h_{2}<-1\right)$. The contribution to the probability $p(\downarrow \uparrow)$ is given by the sector $\left(h_{1}<-1, h_{2}>-2 / 3\right)$. And finally the contribution to the probability $p(\downarrow \downarrow)$ is given by the sector $\left(h_{1}<-2 / 3, h_{2}<-2 / 3\right)$, which overlaps with the sector corresponding to $p(\uparrow \uparrow)$. Thus in the square $\left(-1<h_{1}<-2 / 3 ;-1<h_{2}<-2 / 3\right)$ we are facing a kind of frustration (Figure 5).

One can propose two ways out of this situation. First of all, one can, of course, compare the energies of the two configurations, and then, if the spin orientations always correspond to the ground state, the ambiguity will be lifted (the configuration with both spins "up" has lower energy in the triangle above the dotted line inside the "frustrated square" in Figure 5, and the one with both spins "down" has lower energy below the dotted line). However, if we keep in mind the scenario in which in the course of variations of the external parameter $h$ each spin just follow the direction of the local field, while all thermally activated "jumps over barriers" are suppressed, then we have to conclude that inside the "frustrated" region the orientation of the two spins must depend on the history. Namely, for every point in the plane $\left(h_{1}, h_{2}\right)$ we can assign the "trajectory" which would demonstrate where this particular values of the random fields came from. For instance, in the situation when we study the evolution of the system for increasing value of $h$, (starting from zero) a particular point $\left(h_{1}, h_{2}\right)$ has the "trajectory" which is the straight line connecting this point with the 


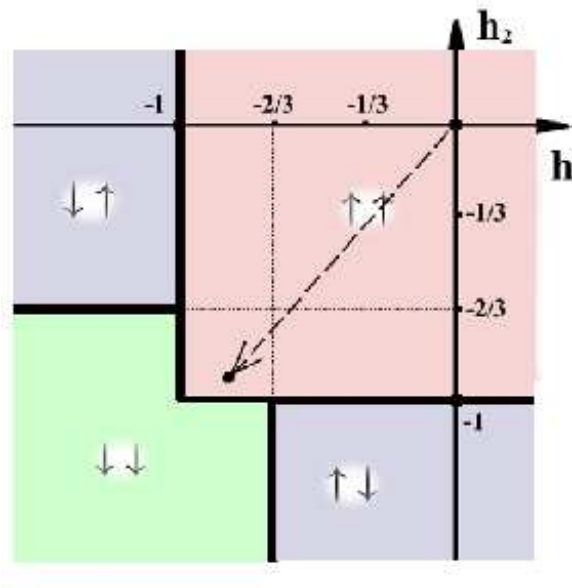

(a)

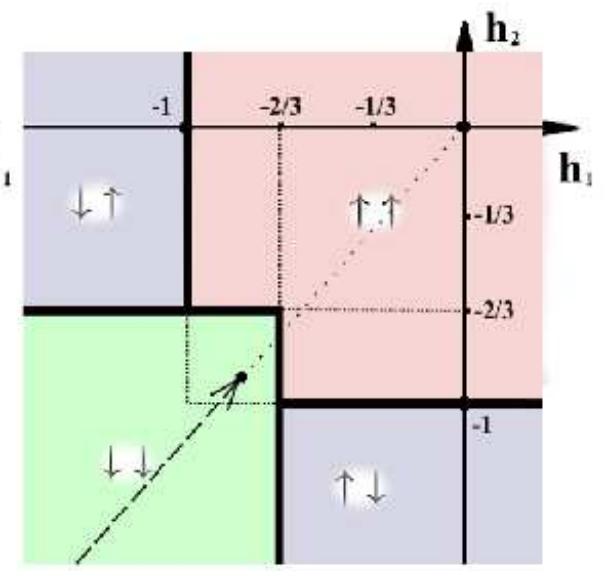

(b)

Figure 6: The orientation diagram of two neighboring spins for the case when all their $(5+5)$ nearest neighbors are directed "up": (a) at increasing and (b) at decreasing strength $h$ of the random fields

origin. In the opposite case of decreasing $h$ (starting from infinity), the "trajectory" of the point $\left(h_{1}, h_{2}\right)$ is the straight line which comes from infinity and directed towards the origin.

Following these rules of the game the ambiguity of the spin orientations is lifted provided one fixes which process is actually under study: increasing or decreasing of $h$. In the study of the ferromagnetic $\rightarrow$ paramagnetic transition (increasing $h$ ) one can easily note that the "trajectory" to any point inside the "frustrated" square $\left(-1<h_{1}<-2 / 3 ;-1<h_{2}<-2 / 3\right)$ comes from the region where both spins, $\sigma_{1}$ and $\sigma_{2}$, are directed "up". Thus, in this case the square "belong" to the probability $p(\uparrow \uparrow)$ (Figure 6(a)). In the reversed process of decreasing $h$ (the paramagnetic $\rightarrow$ ferromagnetic transition) the only way to arrive inside this square is to come from the region where both spins are "down", and in this case the square belong to the probability $p(\downarrow \downarrow)$ (Figure 6(b)).

For better understanding how it works let us consider other examples. In the case when all 10 surrounding spins are directed "down", the "orientation diagram" for the spins $\sigma_{1}$ and $\sigma_{2}$ is represented in Figure 7. Here the "frustrated square" is located in the region $\left(2 / 3<h_{1}<1 ; 2 / 3<h_{2}<1\right)$. Now, at increasing $h$ one arrives into this square from the region where both spins are "down" and therefore in this case it belongs to the probability $p(\downarrow \downarrow)$ (Figure 7(a)). Apparently, in the reversed process of decreasing $h$ the square belong to the probability $p(\uparrow \uparrow)$ (Figure $7(\mathrm{~b})$ ).

Let us consider the configuration in which all 5 neighbors of the spin $\sigma_{1}$ are "up", while all 5 neighbors of the spin $\sigma_{2}$ are "down". Using eqs.(36) one can easily build the corresponding orientation diagram (Figure 8). We see that in this case both at increasing and decreasing $h$ one can arrive into the left-down triangle of the "frustrated square" only by passing the region where both spins are "down". Similarly, both at increasing and decreasing $h$ one can arrive into the right-up triangle only by passing the region where both spins are "up".

Finally, let us consider the configuration in which all 5 neighbors of the spin $\sigma_{1}$ are "up", and only 4 neighbors of the spin $\sigma_{2}$ are "down". Here the division of the "frustrated square" between $p(\uparrow \uparrow)$ and $p(\downarrow \downarrow)$ is somewhat more complicated (Figure 9). At increasing $h$ one arrives into the section of the square below the line $h_{2}\left(h_{1}\right)=-\frac{1}{2} h_{1}$ by passing through the region where both spins are "down" and one arrives into the section above this line by passing through the region where both spins are "up" (Figure 9(a)). On the other hand, at decreasing $h$ one arrives into the section of the square below the line $h_{2}\left(h_{1}\right)=-\frac{2}{3} h_{1}$ by passing through the region where both spins are "down" and one arrives into the section above this line by passing through the region where both spins are "up" (Figure 9(b)).

Now, with some patience and perseverance one can construct the structure of the frustrated squares for all 36 "analytically different" (in terms of eqs.(36)) configurations of the surrounding spins. The resulting orientation diagrams for increasing and decreasing $h$ are shown in Figures 10 and 11 correspondingly. 


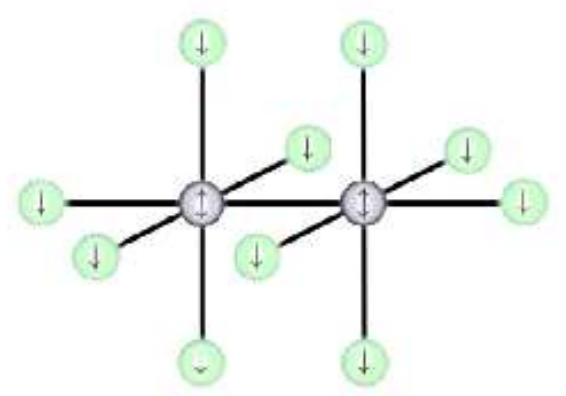

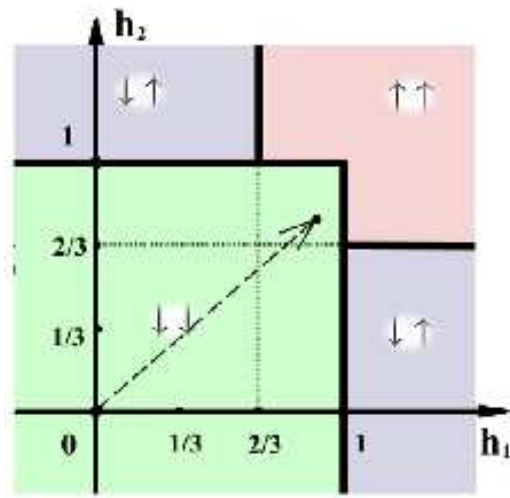

(a)

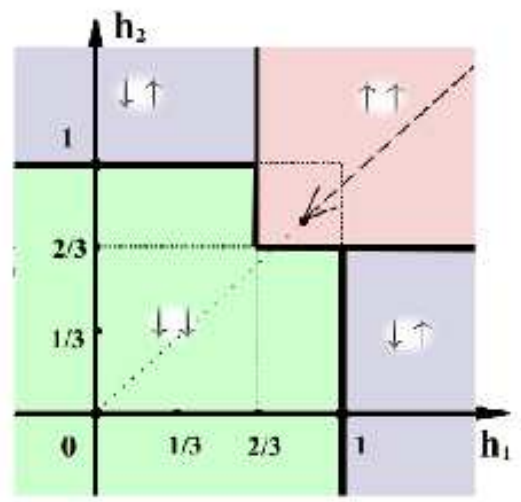

(b)

Figure 7: The orientation diagram of two neighboring spins for the case when all their $(5+5)$ nearest neighbors are directed "down": (a) at increasing and (b) at decreasing strength $h$ of the random fields

Finally, after the regions corresponding to "up-up" and "up-down" orientations of the spins $\sigma_{1}$ and $\sigma_{2}$ are unambiguously defined, the derivation of the equations for the probabilities $p(\uparrow \uparrow)(x, h)$ and $p(\uparrow \downarrow)(x, h)$ is straitforward (although slightly cumbersome). The explicit forms of the equation (35) for increasing and for decreasing $h$ is given in the Appendix.

The solution of these equations in terms of the ferromagnetic order parameter $m(h)$ is represented in Figure 12. We see that the behavior of the magnetization exhibits clear hysteresis phenomenon.

\section{Discussion}

In this paper the zero-temperature phase transition has been studied under the assumption that at any variations of the strength $h$ of the random fields the transformations of the spin configurations go under constrain that all thermally activated spin flips are suppressed. It should be stressed that this situation is essentially different from the true equilibrium phase transition where at any given $h$ the system is supposed to be in the ground state (and which, in my view, is experimentally inaccessible, et least in the low-temperature limit).

Comparing two possible scenarios of the order-disorder phase transition: continuous (the second order) and discontinuous (the first order), one should note that unlike the continuous transition characterized by the divergence of the correlation length, at the first-order phase transition the correlation length remains finite. Thus, admitting that the transition is discontinuous one can hope that a theory which takes into account spin-spin correlations only at a limited scale (e.g. of the order of the lattice spacing) still would give qualitatively correct description of the phase transition.

Usual mean-field theory is useless here because it does not take into account spin-spin correlations at all. On the other hand, it has been demonstarted in this paper that even rather limited improvement of the meanfield approach, which takes into account two nearest neighbor spins correlations, produces rather dramatic effect on the scenario of the phase transition. After this modification the value of the ferromagnetic order parameter $m(h)$ as the function of the strength of the random fields becomes history dependent exhibiting clear hysteresis phenomenon (Figure 12). 

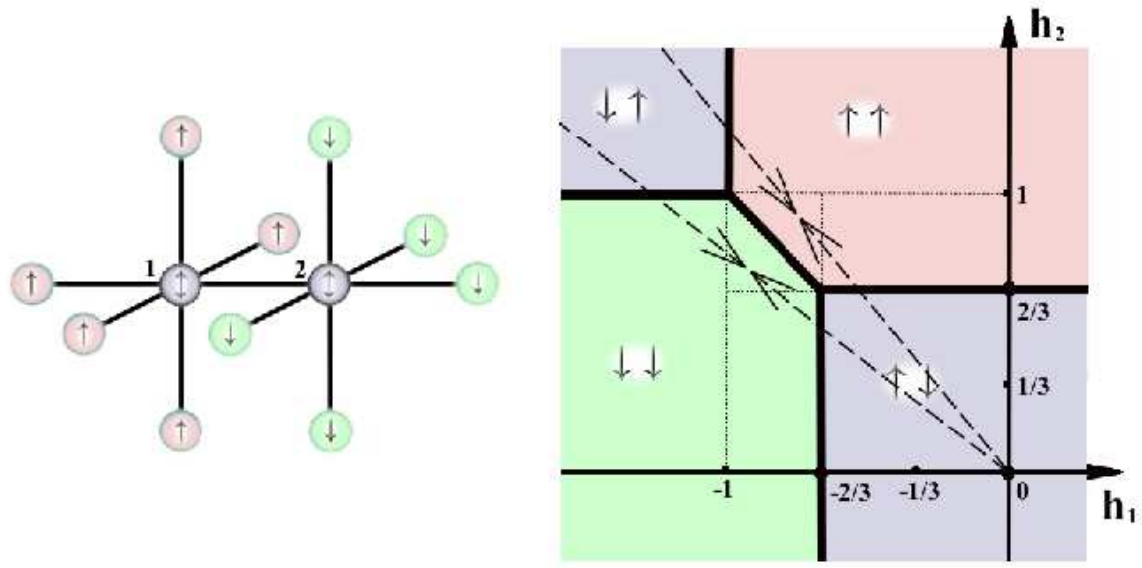

Figure 8: The orientation diagram of two neighboring spins for the case when all 5 neighbors of the spin $\sigma_{1}$ are directed "up", while all 5 neighbors of the spin $\sigma_{2}$ are directed "down"

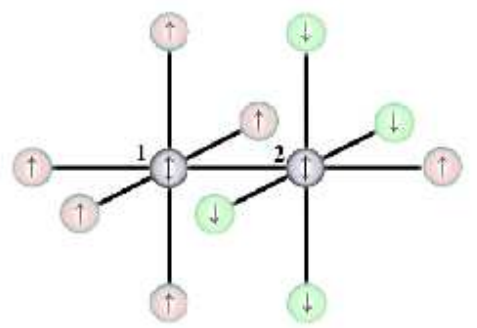

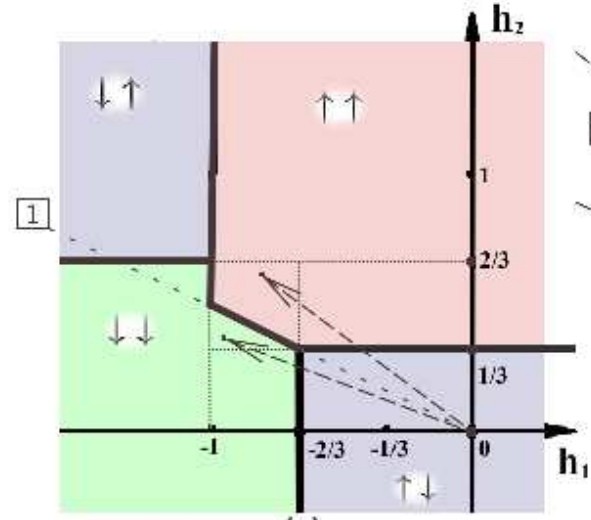

(a)

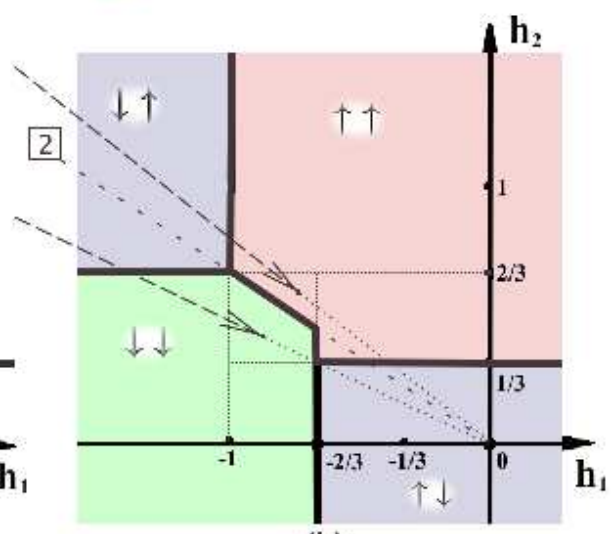

(b)

Figure 9: The orientation diagram of two neighboring spins for the case when all 5 neighbors of the spin $\sigma_{1}$ are directed "up", and 4 neighbors of the spin $\sigma_{2}$ are directed "down": (a) at increasing and (b) at decreasing strength $h$ of the random fields. The lines are: [1] $h_{2}\left(h_{1}\right)=-(1 / 2) h_{1} ;[2] h_{2}\left(h_{1}\right)=-(2 / 3) h_{1}$ 


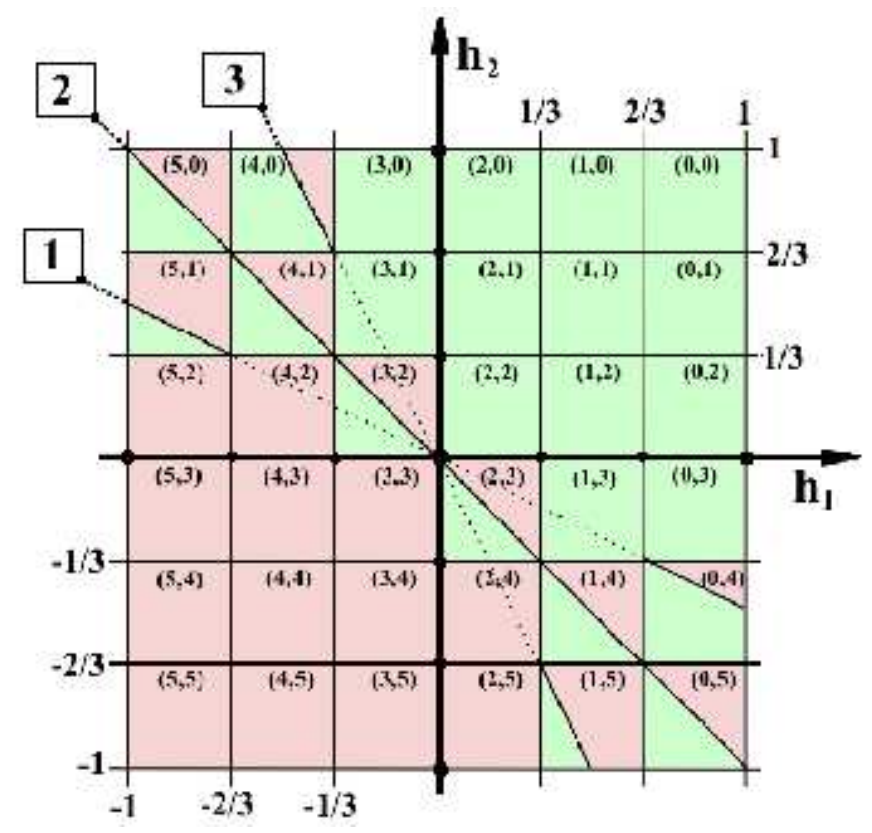

Figure 10: The structure of the "frustrated squares" at increasing strength $h$ of the random fields. The notation $(m, n)$ inside the square indicate that it corresponds to the configuration with $m$ neighbors of the spin $\sigma_{1}$ and $n$ neighbors of the spin $\sigma_{2}$ are directed "up". Rose regions corresponds to the state with both spins $\sigma_{1}$ and $\sigma_{2}$ are directed "up". Green regions are the ones where both spins $\sigma_{1}$ and $\sigma_{2}$ are directed "down". The lines are: [1] $h_{2}\left(h_{1}\right)=-(1 / 2) h_{1} ;[2] h_{2}\left(h_{1}\right)=-h_{1} ;[3] h_{2}\left(h_{1}\right)=-2 h_{1}$.

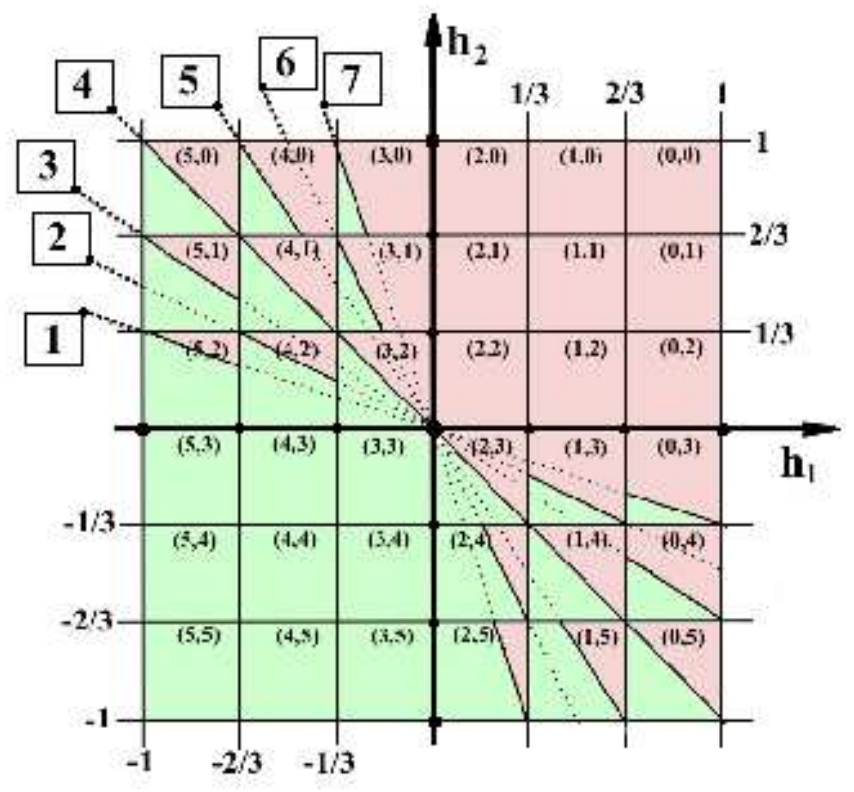

Figure 11: The structure of the "frustrated squares" at decreasing strength $h$ of the random fields. Notations are the same as in Figure 10. The lines are: [1] $h_{2}\left(h_{1}\right)=-(1 / 3) h_{1} ;[2] h_{2}\left(h_{1}\right)=-(1 / 2) h_{1} ;[3] h_{2}\left(h_{1}\right)=$ $-(2 / 3) h_{1} ;[4] h_{2}\left(h_{1}\right)=-h_{1} ;[5] h_{2}\left(h_{1}\right)=-(3 / 2) h_{1} ;[6] h_{2}\left(h_{1}\right)=-2 h_{1} ;[7] h_{2}\left(h_{1}\right)=-3 h_{1}$ 


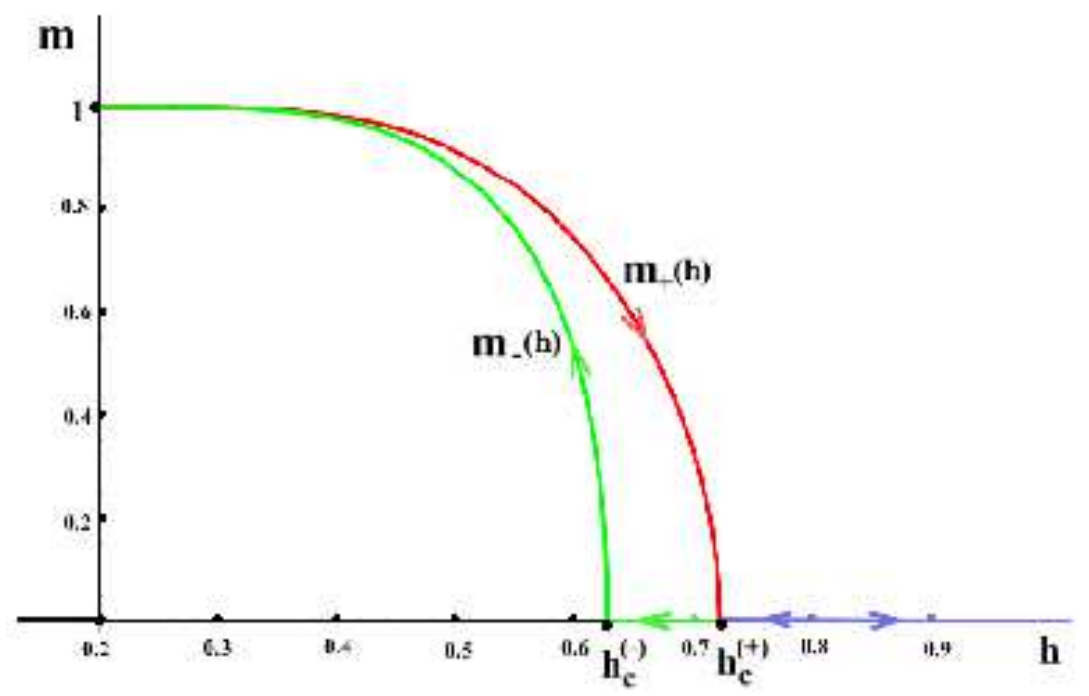

Figure 12: Ferromagnetic order parameter $m(h)$ at increasing (red line) and decreasing (green line) strength $h$ of the random fields. $h_{c}^{(+)} \simeq 0.722 ; h_{c}^{(-)} \simeq 0.622$.

Unfortunately in the framework of the present theory it is still difficult to to make a definite conclusion about the nature of the phase transition. The existence of the hysteresis loop (together with the clear understanding of the physical mechanism in its origin) is the strong argument in favor of the first-order phase transition. On the other hand, each of the curves $m_{+}(h)$ (at increasing $h$ ) and $m_{-}(h)$ (at decreasing $h$ ) in the Figure 12 demonstrates the continuous transition which, of course, makes no sense, because at such transition the correlation length diverges, while the present theory takes into account the correlations only of the order of the lattice spacing. In this sense the present theory is not self-consistent, and it would be reasonable to expect that the presence of the continuous transitions at $h_{c}^{( \pm)}$is not more than just an artifact of the proposed approach. These issues require further detailed studies.

\section{APPENDIX}

Let us denote by $p_{+}(\uparrow \uparrow)$ and $p_{-}(\uparrow \uparrow)$ the probabilities for the two spins $\sigma_{1}$ and $\sigma_{2}$ to be both "up" at increasing and at decreasing variations of $h$ correspondingly. Note that the probability $p(\uparrow \downarrow)$ is the same in both (increasing and decreasing) cases. Then, using the orientation diagrams shown in Figures 10 and 11 (after some work) we get: 


$$
\begin{aligned}
& p_{+}(\uparrow \uparrow)(x, h)=P_{6}(h) P_{6}(h) x^{10}+10 P_{6}(h) P_{5}(h) x^{9}(1-x)^{1} \\
& +\left[20 P_{6}(h) P_{4}(h)+25 P_{5}(h) P_{5}(h)\right] x^{8}(1-x)^{2}+\left[20 P_{6}(h) P_{3}(h)+100 P_{5}(h) P_{4}(h)\right] x^{7}(1-x)^{3} \\
& +\left[10\left[P_{6}(h) P_{2}(h)-D_{51}^{(+)}(h)\right]+100 P_{5}(h) P_{3}(h)+100 P_{4}(h) P_{4}(h)\right] x^{6}(1-x)^{4} \\
& +\left[2\left[P_{6}(h) P_{1}(h)-0.5\left(P_{6}(h)-P_{5}(h)\right)\left(P_{1}(h)-P_{0}(h)\right)\right]+50\left[P_{5}(h) P_{2}(h)-0.5\left(P_{5}(h)-P_{4}(h)\right)\left(P_{2}(h)-P_{1}(h)\right)\right]\right. \\
& \left.+200\left[P_{4}(h) P_{3}(h)-0.5\left(P_{4}(h)-P_{3}(h)\right)\left(P_{3}(h)-P_{2}(h)\right)\right]\right] x^{5}(1-x)^{5} \\
& +\left[10\left[P_{5}(h) P_{1}(h)-\left(P_{5}(h)-P_{4}(h)\right)\left(P_{1}(h)-P_{0}(h)\right)+D_{40}^{(+)}(h)\right]+100\left[P_{4}(h) P_{2}(h)-\left(P_{4}(h)-P_{3}(h)\right)\left(P_{2}(h)-P_{1}(h)\right)\right]\right. \\
& \left.+100\left[P_{3}(h) P_{3}(h)-\left(P_{3}(h)-P_{2}(h)\right)\left(P_{3}(h)-P_{2}(h)\right)\right]\right] x^{4}(1-x)^{6} \\
& +\left[20\left[P_{4}(h) P_{1}(h)-\left(P_{4}(h)-P_{3}(h)\right)\left(P_{1}(h)-P_{0}(h)\right)\right]+100\left[P_{3}(h) P_{2}(h)-\left(P_{3}(h)-P_{2}(h)\right)\left(P_{2}(h)-P_{1}(h)\right)\right]\right] x^{3}(1-x)^{7} \\
& +\left[20\left(P_{3}(h) P_{1}(h)-\left(P_{3}(h)-P_{2}(h)\right)\left(P_{1}(h)-P_{0}(h)\right)\right)+25\left(P_{2}(h) P_{2}(h)-\left(P_{2}(h)-P_{1}(h)\right)\left(P_{2}(h)-P_{1}(h)\right)\right)\right] x^{2}(1-x)^{8} \\
& +10\left[P_{2}(h) P_{1}(h)-\left(P_{2}(h)-P_{1}(h)\right)\left(P_{1}(h)-P_{0}(h)\right)\right] x^{1}(1-x)^{9} \\
& +\left[P_{1}(h) P_{1}(h)-\left(P_{1}(h)-P_{0}(h)\right)\left(P_{1}(h)-P_{0}(h)\right)\right](1-x)^{10} \\
& p_{-}(\uparrow \uparrow)(x, h)= \\
& =\left[P_{6}(h) P_{6}(h)-\left(P_{6}(h)-P_{5}(h)\right)\left(P_{6}(h)-P_{5}(h)\right)\right] x^{10}+10\left[P_{6}(h) P_{5}(h)-\left(P_{6}(h)-P_{5}(h)\right)\left(P_{5}(h)-P_{4}(h)\right)\right] x^{9}(1-x)^{1} \\
& +\left[20\left[P_{6}(h) P_{4}(h)-\left(P_{6}(h)-P_{5}(h)\right)\left(P_{4}(h)-P_{3}(h)\right)\right]+25\left[P_{5}(h) P_{5}(h)-\left(P_{5}(h)-P_{4}(h)\right)\left(P_{5}(h)-P_{4}(h)\right)\right]\right] x^{8}(1-x)^{2} \\
& +\left[20\left[P_{6}(h) P_{3}(h)-\left(P_{6}(h)-P_{5}(h)\right)\left(P_{3}(h)-P_{2}(h)\right)+D_{52}^{(-)}(h)\right]\right. \\
& \left.+100\left[P_{5}(h) P_{4}(h)-\left(P_{5}(h)-P_{4}(h)\right)\left(P_{4}(h)-P_{3}(h)\right)\right]\right] x^{7}(1-x)^{3} \\
& +\left[10\left[P_{6}(h) P_{2}(h)-\left(P_{6}(h)-P_{5}(h)\right)\left(P_{2}(h)-P_{1}(h)\right)+D_{51}^{(-)}(h)\right]\right. \\
& +100\left[P_{5}(h) P_{3}(h)-\left(P_{5}(h)-P_{4}(h)\right)\left(P_{3}(h)-P_{2}(h)\right)+D_{42}^{(-)}(h)\right] \\
& \left.+100\left[P_{4}(h) P_{4}(h)-\left(P_{4}(h)-P_{3}(h)\right)\left(P_{4}(h)-P_{3}(h)\right)\right]\right] x^{6}(1-x)^{4} \\
& +\left[2\left[P_{6}(h) P_{1}(h)-0.5\left(P_{6}(h)-P_{5}(h)\right)\left(P_{1}(h)-P_{0}(h)\right)\right]+50\left[P_{5}(h) P_{2}(h)-0.5\left(P_{5}(h)-P_{4}(h)\right)\left(P_{2}(h)-P_{1}(h)\right)\right]\right. \\
& \left.+200\left[P_{4}(h) P_{3}(h)-0.5\left(P_{4}(h)-P_{3}(h)\right)\left(P_{3}(h)-P_{2}(h)\right)\right]\right] x^{5}(1-x)^{5} \\
& +\left[10\left[P_{5}(h) P_{1}(h)-D_{51}^{(-)}(h)\right]+100\left[P_{4}(h) P_{2}(h)-D_{42}^{(-)}(h)\right]+100 P_{3}(h) P_{3}(h)\right] x^{4}(1-x)^{6} \\
& +\left[20\left[P_{4}(h) P_{1}(h)-D_{52}^{(-)}(h)\right]+100 P_{3}(h) P_{2}(h)\right] x^{3}(1-x)^{7} \\
& +\left[20 P_{3}(h) P_{1}(h)+25 P_{2}(h) P_{2}(h)\right] x^{2}(1-x)^{8}+10 P_{2}(h) P_{1}(h) x^{1}(1-x)^{9}+P_{1}(h) P_{1}(h)(1-x)^{10}
\end{aligned}
$$




$$
\begin{aligned}
& p(\uparrow \downarrow)(x, h)=P_{5}(h) P_{0}(h) x^{10}+\left[P_{5}(h) P_{1}(h)+5 P_{4}(h) P_{0}(h)\right] x^{9}(1-x)^{1} \\
& +\left[10 P_{5}(h) P_{2}(h)+25 P_{4}(h) P_{1}(h)+10 P_{3}(h) P_{0}(h)\right] x^{8}(1-x)^{2} \\
& +\left[10 P_{5}(h) P_{3}(h)+50 P_{4}(h) P_{2}(h)+50 P_{3}(h) P_{1}(h)+10 P_{2}(h) P_{0}(h)\right] x^{7}(1-x)^{3} \\
& +\left[5 P_{5}(h) P_{4}(h)+50 P_{4}(h) P_{3}(h)+100 P_{3}(h) P_{2}(h)+50 P_{2}(h) P_{1}(h)+5 P_{1}(h) P_{0}(h)\right] x^{6}(1-x)^{4} \\
& +\left[P_{5}(h) P_{5}(h)+25 P_{4}(h) P_{4}(h)+100 P_{3}(h) P_{3}(h)+100 P_{2}(h) P_{2}(h)+25 P_{1}(h) P_{1}(h)+P_{0}(h) P_{0}(h)\right] x^{5}(1-x)^{5} \\
& +\left[5 P_{4}(h) P_{5}(h)+50 P_{3}(h) P_{4}(h)+100 P_{2}(h) P_{3}(h)+50 P_{1}(h) P_{2}(h)+5 P_{0}(h) P_{1}(h)\right] x^{4}(1-x)^{6} \\
& +\left[10 P_{3}(h) P_{5}(h)+50 P_{2}(h) P_{4}(h)+50 P_{1}(h) P_{3}(h)+10 P_{0}(h) P_{2}(h)\right] x^{3}(1-x)^{7} \\
& +\left[10 P_{2}(h) P_{5}(h)+25 P_{1}(h) P_{4}(h)+10 P_{0}(h) P_{3}(h)\right] x^{2}(1-x)^{8} \\
& +\left[5 P_{1}(h) P_{5}(h)+5 P_{0}(h) P_{4}(h)\right] x^{1}(1-x)^{9}+P_{0}(h) P_{5}(h)(1-x)^{10}
\end{aligned}
$$

where the functions $P_{k}(h) \quad(k=0, \ldots, 6)$ are defined in eq.(33) and

$$
\begin{aligned}
& D_{51}^{(+)}(h)=\int_{-1}^{-2 / 3} d y_{1} \mathcal{P}_{h}\left(y_{1}\right) \int_{1 / 3}^{-y_{1} / 2} d y_{2} \mathcal{P}_{h}\left(y_{2}\right) \\
& D_{40}^{(+)}(h)=\int_{2 / 3}^{1} d y_{2} \mathcal{P}_{h}\left(y_{2}\right) \int_{-y_{2} / 2}^{-1 / 3} d y_{1} \mathcal{P}_{h}\left(y_{1}\right) \\
& D_{52}^{(-)}(h)=\int_{-1}^{-2 / 3} d y_{1} \mathcal{P}_{h}\left(y_{1}\right) \int_{-y_{1} / 3}^{1 / 3} d y_{2} \mathcal{P}_{h}\left(y_{2}\right) \\
& D_{51}^{(-)}(h)=\int_{-1}^{-2 / 3} d y_{1} \mathcal{P}_{h}\left(y_{1}\right) \int_{-2 y_{1} / 3}^{2 / 3} d y_{2} \mathcal{P}_{h}\left(y_{2}\right) \\
& D_{42}^{(-)}(h)=\int_{-2 / 3}^{-1 / 3} d y_{1} \mathcal{P}_{h}\left(y_{1}\right) \int_{-y_{1} / 2}^{1 / 3} d y_{2} \mathcal{P}_{h}\left(y_{2}\right)
\end{aligned}
$$

The ferromagnetic order parameters $m_{ \pm}(h)$ as the function of $h$ are obtained from the relation

$$
m_{ \pm}(h)=2 x_{ \pm}(h)-1
$$

where $x_{+}(h)$ and $x_{-}(h)$ are the corresponding solutions of the equations

$$
x=p_{ \pm}(\uparrow \uparrow)(x, h)+p(\uparrow \downarrow)(x, h)
$$

\section{References}

[1] T.Nattermann and J.Villain, Phase Transitions, 11, 5 (1988);

D.P.Belanger and A.P.Young, Journal of Magnetism and Magnetic Materials 100, 272 (1991);

D.P.Belanger Experiments on the Random Field Ising Model in Spin Glasses and Random Fields, ed. by A.P.Young (World Scientific, Singapore, 1998)

T.Nattermann Theory of the Random Field Ising Model in Spin Glasses and Random Fields, ed. by A.P.Young (World Scientific, Singapore, 1998)

[2] V.Jaccarino and A.R.King Physica A 163, 291 (1990)

[3] A.I.Larkin, Sov.Phys. JETP 31, 784 (1970) 
[4] Y.Imry and S.-K.Ma, Phys.Rev.Lett. 35, 1399 (1975)

[5] J.Imbrie, Phys.Rev.Lett. 53, 1747 (1984);

J.Z.Imbrie, Commun.Math.Phys. 98, 145 (1985);

J.Bricmont and A.Kupiainen, Phys.Rev.Lett. 59, 1929 (1987)

[6] A.Aharony, Y.Imry and S.-K.Ma, Phys.Rev.Lett. 37, 1364 (1976);

A.P.Young, J.Phys. A 10, L257 (1977);

G.Parisi and N.Sourlas, Phys.Rev.Lett. 43, 774 (1979);

G.Parisi, Quantum Field Theory and Quantum Statistics, (Bristol, Adam Hilger, 1987)

[7] G.Parisi, Proceedings of Les Houches 1982, Session XXXIX, edited by J.B.Zuber and R.Stora (North Holland, Amsterdam, 1984);

[8] M.Guagnelli, E.Marinari and G.Parisi, J.Phys. A, 26, 5675 (1993).

[9] T.Schneider and E.Pytte, Phys.Rev. B 15, 1519 (1977)

[10] A.Aharony, Phys.Rev. B 18, 3318 (1978)

[11] G.Grinstein Phys.Rev.Lett. 37, 944 (1976);

A.J.Bray and M.A.Moore J.Phys. C, 18, L927 (1985);

D.S.Fisher, Phys.Rev.Lett. 56, 416 (1986)

[12] M.Mezard and G.Parisi, J.Phys. A, 23, L1229 (1990)

[13] G.Parisi, J.Phys. A, 13, 1887 (1980)

[14] M.Mezard and A.P.Young, Europhys.Lett. 18, 653 (1992)

[15] M.Mezard and R.Monasson, Phys.Rev. B 50, 7199 (1994)

[16] C.De Dominicis, H.Orland and T.Temisvari, J. de Physique I 5, 987 (1995)

[17] E.Brezin and C.De Dominicis, Europhys. Lett. 44, 13 (1998);

E.Brezin and C.De Dominicis, Eur. Phys. J. 19, 467 (2001)

[18] G.Parisi and Vik.S.Dotsenko, J.Phys. A 25, 3143 (1992)

[19] Vik.S.Dotsenko J.Phys. A 27, 3397 (1994)

[20] Vik.S.Dotsenko, J.Stat.Phys., 122 (2), 197 (2006)

[21] Vik.S.Dotsenko, Physica A, 361, 463 (2006)

[22] Vik.S.Dotsenko, J.Stat.Mech. P06003 (2006)

[23] P. Le Doussal and K.Wiese, Phys.Rev.Lett. 96, 197202 (2006);

G.Tarjus and M.Tissier, Phys.Rev.Lett. 93, 267008 (2004)

[24] A.Houton, A.Khurana anf F.J.Seco, Phys.Rev.Lett. 55, 856 (1985)

[25] M.Goffman, J.Adler, A.Aharony, A.B.Harris and M.Schwartz, Phys.Rev. B 54, 364 (1996)

[26] A.P.Young and M.Nauenberg, Phys.Rev.Lett. 54, 2429 (1985)

[27] A.T.Ogielski and D.A.Huse, Phys.Rev.Lett. 56, 1298 (1986)

[28] A.T.Ogielski, Phys.Rev.Lett. 57, 1251 (1986)

[29] H.Rieger and A.P.Young, J.Phys. A 26, 5279 (1993);

H.Rieger, Phys.Rev. B 52, 6659 (1995) ;

M.E.J.Newman and G.T.Barkema, Phys.Rev. E 53, 393 (1996)

[30] J.Machta, M.E.J.Newman and L.B.Chayes, Phys.Rev. E 62, 393 (2000) 
[31] M.S.Chao and J.Machta, Phys.Rev. B 48, 3177 (1993);

[32] A.Falicov, N.Berker and S.R. McKay, Phys.Rev. B 51, 8266 (1995)

[33] J.-C.Angles d'Auriac and N.Sourlas Europhys.Lett. 39, 473 (1997); N.Sourlas, Comput.Phys.Commun. 121, 184 (1999)

[34] M.R.Swift, A.J.Bray, A.Maritan, M.Cieplak and J.R.Banavar, Europhys.Lett. 38, 273 (1997); A.K.Hartmann and U.Nowak, Eur.Phys.J. B 7,105 (1999); A.K.Hartmann and A.P.Young, Phys.Rev. B 64, 214419 (2001)

[35] A.A. Middleton and D.S.Fisher, Phys.Rev. B 65, 134411 (2002)

[36] Y.Wu and J.Machta, Phys.Rev.Lett. 95, 137208 (2005)

[37] M.Itakura, Phys.Rev. B 64, 012415 (2001)

[38] J.Siniva and G.Canright, Phys.Rev. B 64, 094402 (2001)

[39] G.S.Grest, C.M.Soukoulis and K. Levin, Phys.Rev. B 33, 7659 (1986)

[40] A.Maiorano, V.Martin-Mayor, J.J.Ruiz-Lorenzo, A.Tarrancon, Weak first order transition in the threedimensional site-diluted Ising antiferromagnet in a field, Preprint arXiv:0705.1517v2

[41] V.V.Prudnikov and V.N.Borodikhin, JETP, 101, 294 (2005)

[42] G.Parisi and N.Sourlas, Phys.Rev.Lett. 89, 257204 (2002);

A.Malakis and N.G.Fytas, Phys.Rev. E 73, 016109 (2006)

[43] S.Fishman and A.Aharony, J.Phys. C 12, L729 (1979);

J.Cardy, Phys.Rev. B 29, 505 (1984)

[44] H.Yoshizawa, R.A.Cowley, G.Shirane, R.G.Birgeneau, H.J.Guggenheim and H.Ikeda, Phys.Rev.Lett. 48, 438 (1982);

M.Hagen, R.A.Cowley, S.K.Satija, H.Yoshizawa, G.Shirane, R.G.Birgeneau and H.J.Guggenheim, Phys.Rev. B 28, 2602 (1983)

[45] D.P.Belanger, A.R.King, V.Jaccarino and J.L.Cardy, Phys.Rev. B 28, 2552 (1983);

P.Pollak, W.Kleemann and D.P.Belanger, Phys.Rev. B 38, 4773 (1988)

[46] D.P.Belanger, A.R.King and V.Jaccarino, Phys.Rev. B 31, 4538 (1985)

[47] R.J.Birgeneau, R.A.Cowley G.Shirane and H.Yoshizawa Phys.Rev.Lett. 54, 2147 (1985)

[48] C.A.Ramos, A.R.King, V.Jaccarino and S.M.Resende, J.Physique (France) 49 C8-1241 (1988)

[49] F.Ye, L.Zhou, S.Larochelle, L.Lu, D.P.Belanger, M.Greven and D.Lederman, Phys.Rev.Lett. 89, 157202 (2002)

[50] J.P.Hill, T.R.Thurston, R.W.Ervin, M.J.Ramstad and R.J.Birgeneau, Phys.Rev.Lett. 66, 3291 (1991)

[51] R.J.Birgeneau, Q.Feng, Q.J.Harris, J.P.Hill and A.P.Ramirez, Phys.Rev.Lett. 77, 2342 (1996)

[52] P.-Z.Wong and J.W.Cable Phys.Rev. B 28, 5361 (1983);

P.-Z.Wong, Phys.Rev.Lett. 77, 2338 (1996)

[53] J.P.Hill, Q.Feng, Q.J.Harris, R.J.Birgeneau, A.P.Ramirez and A.Cassanho, Phys.Rev. B 55, 356 (1997)

[54] R.J.Birgeneau, Journal of Magnetism and Magnetic Materials 177, 1 (1998)

[55] Z.Slanic, D.P.Belanger and J.A.Fernandez-Baca, Journal of Magnetism and Magnetic Materials 177, 171 (1998)

[56] Z.Slanic, D.P.Belanger and J.A.Fernandez-Baca, Phys.Rev.Lett. 82, 426 (1999) Z.Slanic, D.P.Belanger and J.A.Fernandez-Baca, J.Phys.: Condens.Matter 13, 1711 (2001) 
[57] Z.Slanic and D.P.Belanger, Journal of Magnetism and Magnetic Materials 186, 65 (1998)

[58] M.Aizenman and J.Wehr, Phys.Rev.Lett. 62, 2503 (1989);

K.Uzelac, A.Hasmy and R.Jullien, Phys.Rev.Lett. 74, 422 (1995);

J.Cardy and J.Jacobsen Phys.Rev.Lett. 79, 4063 (1997);

P.N.Timonin, Phys.Rev. B 69, 092102 (2004) 